\title{
Galaxy Disruption as the Origin of Intracluster Light in the Coma Cluster
}

\author{
Michael D. Gregg] \& Michael J. West²
}

\begin{abstract}
Although the existence of a faint background of starlight in the core of the Coma cluster has been well established ${ }^{1,2}$, its origin is uncertain. This vast sea of stars could have formed outside the galaxies, using gas left over from the time of the cluster's birth. Alternatively, it might be the accumulated debris generated by interactions between the galaxies over the lifetime of the cluster ${ }^{3}$. Here we report the discovery of three large, low surface brightness features in the Coma cluster; the most spectacular is a plume-like structure, $130 \mathrm{kpc}$ long, in the cluster's heart. These structures will disperse over the next one to two billion years, thereby enhancing the general background light. If this epoch is typical, we argue that a significant fraction - perhaps even most - of the intracluster light results from a steady accumulation of tidal debris generated during galaxy-galaxy and galaxy-cluster interactions.
\end{abstract}

To Appear in Nature, December 10, 1998

\footnotetext{
${ }^{1}$ Physics Department, University of California, Davis, California 95616, and Institute for Geophysics and Planetary Physics, Lawrence Livermore National Laboratory, L-413 Livermore, California 94550, USA

${ }^{2}$ Department of Astronomy and Physics, Saint Mary's University, Halifax, Nova Scotia B3H 3C3, Canada
} 
We have been using the Kitt Peak Burrell Schmidt 0.6 metre telescope to conduct a multicolour survey of the Coma cluster and its environs over a $3^{\circ} \times 3^{\circ}$ area. The CCD on the Schmidt has $2^{\prime \prime}$ pixels and a field of view of $1.1^{\circ}$. In March of 1996, we took a series of images centred midway between NGC4874 and 4889, the supergiant elliptical galaxies which dominate the centre of the Coma cluster.

Inspection of the final R-band image (Fig. 1) reveals the presence of three large, low surface brightness (LSB) features. Their locations and approximate sizes are indicated by the heavy solid lines labeled '1', '2', and '3' in Fig. 1. All of these objects can be seen in the $\mathrm{B}, \mathrm{V}$, and I frames as well, confirming their reality. All are much larger and more prominent than the recently reported slim arc of tidal debris in the Coma cluster $^{4}$ (no. 4 in Fig. 1). Given the myriad images of the Coma cluster that have been obtained over the years, it is surprising that these features have escaped notice until now. There are at least two instances in the literature of images that show one or more of them clearly ${ }^{5,6}$, but without any comment by the authors. To isolate the low surface brightness (LSB) features, we digitally 'cleaned' portions of the images to remove superposed galaxies and stars by interpolation with background replacement (Fig. 2 and $3)$.

$\boldsymbol{L S B}$ object no. 1 The most striking of the features is a plume-like structure adjacent to NGC4874 (Figs 1 and 2). It extends for at least $4.5^{\prime}$ and varies from $30^{\prime \prime}$ to $60^{\prime \prime}$ in width; it may well extend even farther to the west, past the large elliptical galaxy NGC4864. Adopting a distance of $100 \mathrm{Mpc}$ to Coma, the plume

\footnotetext{
${ }^{3}$ Kitt Peak National Observatory, National Optical Astronomy Observatory, is operated by the Association of Universities for Research in Astronomy, Inc. (AURA), under cooperative agreement with the National Science Foundation. Observations made with the Burrell Schmidt telescope of the Warner and Swasey Observatory, Case Western Reserve University.
}

is $\sim 130 \mathrm{kpc}$ in length and varies from 15 to $30 \mathrm{kpc}$ wide. The integrated apparent magnitude is $R=15.6 \pm 0.1$, though it may contain more material outside our adopted borders. The mean R-band surface brightness is $\mu_{R}=25.7$ $\mathrm{mag} / \operatorname{arcsec}^{2}$. The colours of the plume are consistent with those of normal elliptical galaxies of intermediate brightness, although the photometric uncertainties and the removal of superposed objects leave open the possibility of significant star formation in the LSB material.

$\boldsymbol{L S B}$ object no. 2 This feature is a pool of diffuse light adjacent to a group of galaxies including IC3957, IC3959, and IC3963 (upper panel of Fig. 3.) It is located near one end of a broader, extended swath of even lower surface brightness cluster background light ${ }^{5}$, linking it to NGC4874. This association makes it likely that the feature is within the Coma cluster core. The pool of material has a roughly circular distribution, $\sim 80^{\prime \prime}$ in diameter $(\sim 40 \mathrm{kpc})$. Its colours and surface brightness are similar to those of the plume, with a total integrated magnitude 0.6 fainter.

LSB object no. 3 The third LSB feature is quite chaotic in appearance (lower panel, Fig. 3). The lowest surface brightness portions of this object form a bridge to the outskirts of the Coma member galaxy NGC4911, where there is additional low surface brightness material (inset, Fig. 3); the association places the feature in the Coma cluster. Several small knots are visible in the highest surface brightness portion, suggestive of star formation; however, its colours are not particularly blue as would be expected in that case. Its overall photometric properties are similar to the other LSB features.

$\boldsymbol{L S B}$ object no. 4 has already been described in detail by Trentham \& Mobasher" as a "giant low surface brightness arc of length $\sim 80$ kpc." From our low resolution Schmidt images, we cannot confidently recognize this feature as a tidal arc rather than a curious alignment of faint galaxies, nor can we analyse it in any detail. Its total length is $180^{\prime \prime}$ with a mean B sur- 
face brightness of $<26.5 \mathrm{mag} \operatorname{arcsec}^{-2}$ (ref. 4); in our R-band image, its average width is $\sim 5^{\prime \prime}$. Assuming typical galaxy colours, we estimate the integrated $\mathrm{R}$ magnitude as about 18 , roughly 5-10 times fainter than the other LSB features reported here, with a comparable mean surface brightness.

The LSB features all appear to be non-equilibrium configurations of stars deep within the gravitational potential well of the Coma cluster. We believe that they are best interpreted as transient features produced either by galaxygalaxy interactions or by stripping of galaxies by the global cluster tidal field. Coma's large $\sim$ $1,000 \mathrm{~km} \mathrm{~s}^{-1}$ velocity dispersion would seem to preclude strong galaxy-galaxy interactions since the energy transferred between systems is roughly proportional to the time spent in close proximity; typical high-speed encounters between galaxies will not produce significant disturbances ${ }^{7}$. Yet the presence of these LSB objects shows that Coma's galaxy population is undergoing vigorous dynamical evolution. There is now ample evidence from both optical and X-ray observations that the Coma cluster is a dynamically young system with several components in the throes of merging ${ }^{2,8-12}$. The chaotic nature of subcluster mergers and the relatively low internal velocity dispersions of constituent subgroups, such as the recently identified "galaxy aggregates" in Coma ${ }^{13}$, provide opportunities for lower speed encounters, producing both galaxy-galaxy and galaxy-cluster tidal interactions.

Linear features such as the plume are routinely produced in numerical simulations of galaxy tidal interactions ${ }^{14,15}$, although the plume's origin is hard to pinpoint from our data. It may be the result of interaction between the two ellipticallike nuclei embedded near its centre, though their large velocity difference $(>1,100 \mathrm{~km} \mathrm{~s}-1)^{16}$ makes it unlikely that they could have generated such a long tidal feature while remaining apparently close together ${ }^{14}$. Tides acting in the outer regions of NGC4864 (the large elliptical galaxy located near the plume's western end) could also produce the plume. Another possibility, perhaps less likely, is that the plume is the remains of a galaxy being completely disrupted by the global tidal field as it plunges into the cluster core, not unlike the dive into Jupiter of comet ShoemakerLevy.

The amorphous structures of the LSB features 2-4 are not typical of what is seen in simulations, or examples on the sky, of tidal debris; nevertheless, tidal interaction is a natural explanation. LSB object no. 2 may be the product of strong tidal interaction within the subgroup containing IC3959 and IC3963; their velocity difference is only $240 \mathrm{~km} \mathrm{~s}^{-1}$ (ref. 16). NGC4911 and LSB object no. 3 lie within a $\sim 1 \mathrm{Mpc}$-long ridge of heightened X-ray emission (see Fig. 1) which may have been produced by tidal disruption of a galaxy group on its recent passage through the

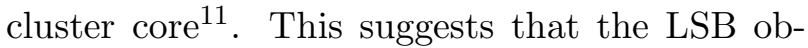
ject no. 3 may have originated during the same dynamical interaction that has brightened the Xray emission. It could be the remains of a galaxy that has completely disrupted or it may be material that has been stripped from the outskirts of NGC4911. The Trentham \& Mobasher object is less clearly associated with cluster member galaxies, but it too is best explained by tidal processes ${ }^{4}$.

With four examples of rather severe tidal interactions, one should expect many more less spectacular encounters to be taking place. The coarse resolution Schmidt images, however, may be incapable of revealing them as such; LSB feature no. 4 is detected, but its nature is not apparent. Our data suggest that there are many weaker tidal interactions and galaxy disturbances taking place in Coma, but finer images which reach at least as deep will be necessary to analyse them. If evidence for weaker interactions is found, it will help account for the dearth of low density dwarf galaxies in Coma's core ${ }^{17}$, as such galaxies are most susceptible to tidal disruption by the cluster's strong gravitational field, and 
also the anti-correlation between the luminosity of the brightest galaxy in a cluster and the size of the dwarf galaxy population. ${ }^{18}$.

The LSB features will gradually disperse, either blending into the existing background of diffuse intracluster material or accreting onto the giant galaxies NGC4889 and NGC4874 at the bottom of Coma's gravitational potential well. All four tidal features are located within $\sim 0.5 \mathrm{Mpc}$ of the cluster center and should dissipate in no more than a few cluster crossing times, about 1 to 2 billion years. If this material contains quickly evolving massive stars or even ongoing star formation, the use of brightest cluster galaxies as standard candles is further complicated. ${ }^{19,20}$ The very highest velocity material will contribute to the population of intergalactic stars and perhaps intergalactic globular clusters as well; evidence of such components may have been detected ${ }^{21,22,23,24}$ in other clusters. If we are viewing Coma at a typical moment in its history, then the presence of at least four large transient star piles indicates that a substantial amount of material could have been liberated from galaxies over the cluster's lifetime; every $\sim 1$ Gyr, the equivalent of a galaxy of Rband absolute magnitude $\mathrm{M}_{R} \approx-19$ is being disrupted and accreted by the cluster core. Integrated over the 10 to 20 billion year history of the Universe, the amount of stellar material added to the pool of intracluster light would be equivalent to an object with $\mathrm{M}_{R} \approx-22$. This is about $20 \%$ of the total luminosity of either NGC4874 or NGC4889, and the interaction rate was almost certainly higher in the past. A significant portion of the mass of the central galaxies may consist of material accreted from tidally dismantled galaxies as the cluster dynamically evolves ${ }^{18,20}$. The LSB objects now in Coma provide a vivid 'snapshot' of this process in action.

\section{REFERENCES}

1. Zwicky, F. Morphological Astronomy p. 48 (Springer-Verlag, Berlin, 1957).
2. Biviano, A. in A New Vision of an Old Cluster: Untangling Coma Berenices (eds Mazure, A., Casoli F., Durret F., \& Gerbal D) 1-8 (World Scientific Publishing, Singapore, 1998).

3. Merritt, D. Relaxation and tidal stripping in rich clusters of galaxies. II - evolution of the luminosity distribution. Astrophys. J. 276, 26-37 (1984).

4. Trentham, N., \& Mobasher, B. The discovery of a giant debris arc in the Coma cluster. Mon. Not. R. Astron. Soc. 293, 53-59 (1998)

5. Welch, G.A., \& Sastry, G.N. Photographic detection of "intergalactic" matter in the Coma cluster. Astrophys. J. 169, L3-L5 (1971).

6. Secker, J., \& Harris, W.E. Dwarf galaxies in the Coma cluster I. detection, measurement and classification techniques Publ. Astron. Soc. Pac. 109, 1364-1376 (1997).

7. Binney, J., Tremaine, S. Galactic Dynamics (Princeton Univ. Press, 1987).

8. Fitchett, M.J., \& Webster, R.L. Substructure in the Coma cluster. Astrophys. J. 317, 653667 (1987).

9. Mellier, Y., Mathez, G., Mazure, A., Chauvineau, B., \& Proust, D. Subclustering and evolution of the Coma cluster. Astron. Astrophys. 199, 67-72 (1988).

10. White, S.D.M., Briel, U.G., \& Henry, J.P. X-ray archaeology in the Coma cluster. Mon. Not. R. Astron. Soc. 261, L8-L12 (1993).

11. Vikhlinin, A., Forman, W., \& Jones, C. Another collision for the Coma cluster. Astrophys. J. 474, L7-L10 (1997).

12. Colless, M. \& Dunn, A.M. Structure and dynamics of the Coma cluster. Astrophys. J. 458, 435-454 (1996).

13. Conselice, C.J., \& Gallagher, J.S. Galaxy aggregates in the Coma cluster. Mon. Not. R. Astron. Soc. 297, L34-L38 (1998).

14. Dubinski, J., Mihos, J.C., \& Hernquist, L. Using tidal tails to probe dark matter halos. 
Astrophys. J. 462, 576-593 (1996).

15. Moore, B., Katz, N., Lake, G., Dressler, A., \& Oemler, A., Jr. Galaxy harassment and the evolution of clusters of galaxies. Nature 379, 613-616 (1996).

16. NASA/IPAC Extragalactic Database, nedwww.ipac.caltech.edu (1998).

17. Thompson, L.A., \& Gregory, S.A. Dwarf galaxies in the Coma cluster. Astron. J. 106, 2197-2212 (1993).

18. Lopez-Cruz, O., Yee, H.K.C., Brown, J.P., Jones, C., \& Forman, W. Are luminous cD halos formed by the disruption of dwarf galaxies? Astrophys. J. 475, L97-L101 (1997).

19. Tinsley, B.M. A First Approximation to the Effect of Evolution on $q_{0}$. Astrophys. J. 173, L93-L97 (1972).

20. Ostriker, J.P., \& Tremaine, S.D. Another evolutionary correction to the luminosity of giant galaxies. Astrophys. J. 202, L113-L116 (1975).

21. Theuns, T. \& Warren, S.J. Intergalactic stars in the Fornax cluster. Mon. Not. R. Astron. Soc. 284, L11-L15 (1997).

22. Ferguson, H.C., Tanvir, N.R., \& von Hippel, T. Detection of intergalactic red-giant-branch stars in the Virgo cluster. Nature 391, 461463 (1998).

23. Ciardullo, R., Jacoby, G.H., Feldmeier, J.J., \& Bartlett, R.E. The planetary nebula luminosity function of M87 and the intracluster stars of Virgo. Astrophys. J. 492, 62-73 (1998).

24. West, M., Cote, P., Jones, C., Forman, W., \& Marzke, R. Intracluster globular clusters. Astrophys. J. 453, L77-L80 (1995).

25. Gregg, M.D. Differential population synthesis of S0 galaxies. I - the data. Astrophys. J. Suppl. 69, 217-232 (1989).

26. Sandage, A., Tammann, G.A. A revised Shapley-Ames catalog of bright galaxies (Carnegie
Inst. of Washington, 1987).

MJW acknowledges a research grant from NSERC of Canada. Part of the work reported here was done at the Institute of Geophysics and Planetary Physics, under the auspices of the U.S. Department of Energy by Lawrence Livermore National Laboratory. We acknowledge use of the NASA/IPAC Extragalactic Database (NED) which is operated by the Jet Propulsion Laboratory, Caltech, under contract with the National Aeronautics and Space Administration.

This 2-column preprint was prepared with the AAS LATEX macros v4.0. 


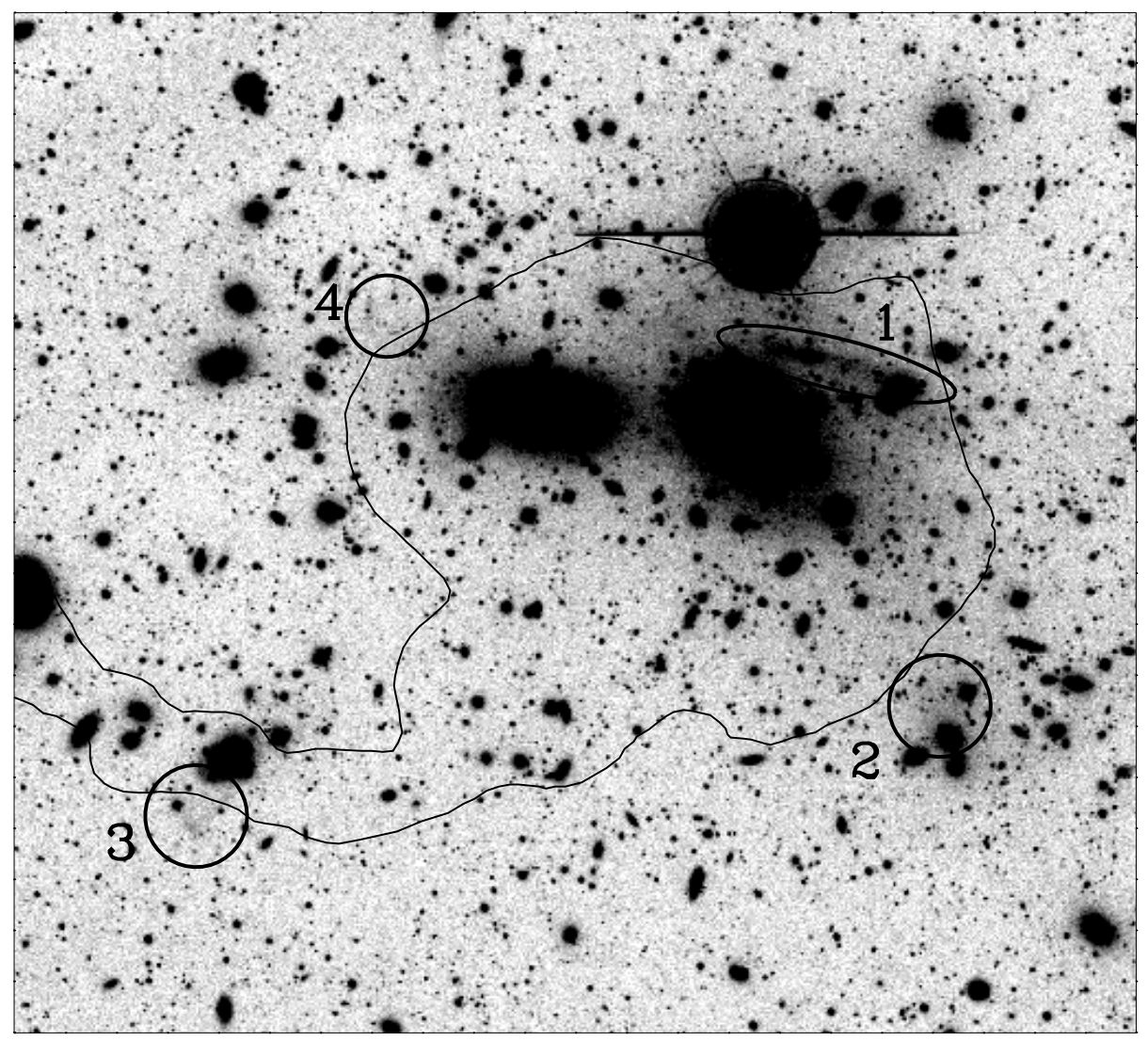

Fig. 1. - R-band image of the Coma cluster core showing the locations and approximate sizes of the low surface brightness features (heavy solid circles labeled 1-4) discussed here. The image is $37^{\prime} \times 33^{\prime}$; north is up, east to the left. Observing conditions were photometric with good seeing, and there was no Moon. The R-band integration totals 75 min.; the B, V, and I band images total 30-45 min. The images were reduced using standard procedures and combined using image masks to remove cosmetic defects on the CCD. Flatfielding using twilight images resulted in peak-to-peak sky flatness of $\sim 2 \%$ in all colours. The thin solid line is one X-ray contour from ROSAT observations ${ }^{11}$ showing the extended ridge which includes LSB object no. 3. 


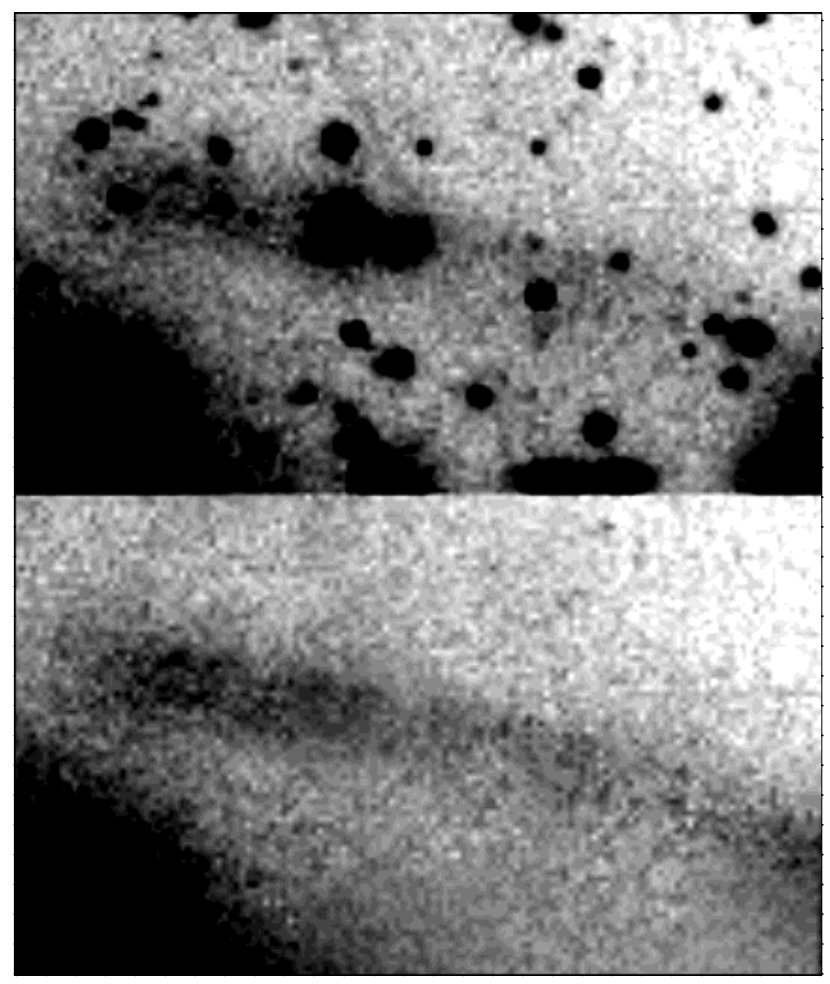

Fig. 2. - The plume feature before (top) and after (bottom) removing foreground objects. North is up, east is to the left. The sloping background from the giant elliptical NGC4874 was fit and removed and the remaining counts from the plume summed to determine a total brightness. The plume is roughly $4^{\prime} .5 \times 1^{\prime}$ in extent, with an integrated R-band magnitude $\mathrm{M}_{R}=15.6$ and mean R-band surface brightness of $\sim 26.3 \mathrm{mag}$ $\operatorname{arcsec}^{-2}$. Adopting a distance of $100 \mathrm{Mpc}$ for the Coma cluster (equivalent to assuming $\mathrm{H}_{\mathrm{o}} \approx 70$ $\mathrm{km} \mathrm{s}^{-1} \mathrm{Mpc}^{-1}$ ), the plume is roughly 130 by 20 kiloparsecs in extent, with an integrated absolute R-band magnitude of -19.4. To estimate the colours, we restricted attention to an area of $80^{\prime \prime} \times 40^{\prime \prime}$ where the surface brightness is highest, near the eastern end. The removal of embedded features was done in a consistent way in all colours. These inclusions may in fact be part of the tidally disrupted material, but it is impossible to determine this with certainty from the low resolution Schmidt images; our conservative approach was to measure only the smooth underlying light belonging to the plume. While the background can be determined to better than $1 \%$, this contributes an uncertainty of 0.1 to 0.2 magnitudes (10 to $20 \%$ ) in the colours because of the faintness of the feature. The plume's colours are $\mathrm{B}-\mathrm{V}=0.93, \mathrm{~V}-\mathrm{R}=0.57$, and $\mathrm{V}-\mathrm{I}=1.17$, consistent with those of a normal elliptical galaxy. For comparison, the normal elliptical NGC3377 ${ }^{24}$ has $\mathrm{B}-\mathrm{V}=0.92, \mathrm{~V}-\mathrm{R}=0.58, \mathrm{~V}-\mathrm{I}=1.20$ and an absolute $\mathrm{R}$ magnitude ${ }^{25}$ of -20.8 . These colours indicate little if any recent star formation associated with the tidal event. This constraint is not strong, however, because the colours have been determined from a restricted area of the feature and the removed, apparent foreground, objects may be star formation activity in the plume. 


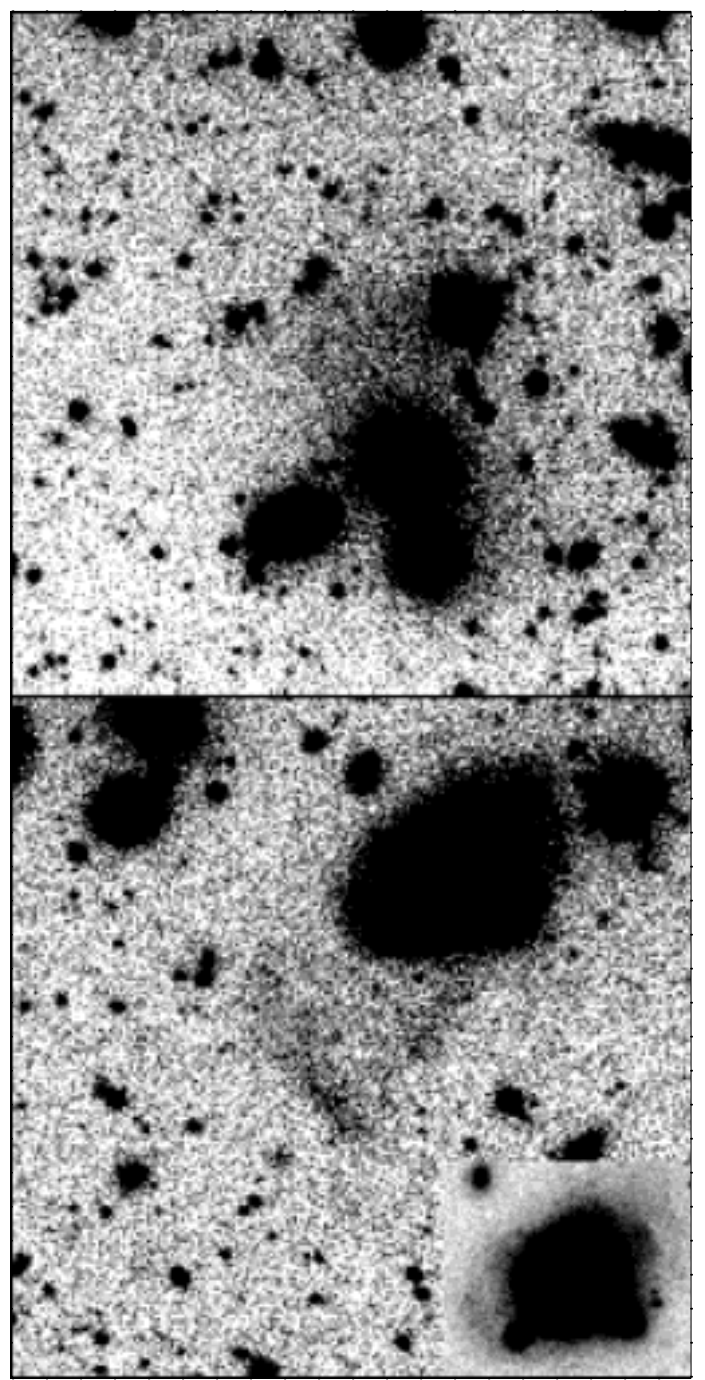

Fig. 3.- LSB Objects no. 2 (upper) and no. 3 after removing foreground stars and galaxies. Each image is $400^{\prime \prime}$ square. The colours and absolute magnitude of no. 2 are $\mathrm{B}-\mathrm{V}=0.90, \mathrm{~V}-\mathrm{R}=0.43, \mathrm{~V}$ $\mathrm{I}=1.06, \mathrm{M}_{R}=-18.8$ and no. 3 are $\mathrm{B}-\mathrm{V}=0.93, \mathrm{~V}$ $\mathrm{R}=0.59, \mathrm{~V}-\mathrm{I}=0.89, \mathrm{M}_{R}=-18.3$. Like the plume feature, these are similar to a normal elliptical galaxy and indicate little if any recent star formation, though here too, the removed foreground objects may in fact be part of the tidal material. The bright object immediately to the northwest of feature no. 2 is a Galactic star. The two objects immediately south of it are IC3959 and IC3963; their low velocity difference of $240 \mathrm{~km}$ $\mathrm{s}^{-1}$ may allow the strong tidal interaction which gives rise to the LSB material. The bright object to the northwest of feature no. 3 is NGC4911, a face-on spiral. The inset in the lower right is a lower contrast display of NGC4911 showing the low surface brightness material encircling its disk and linking it to LSB Object no. 3. NGC4911 has a close S0 companion which is partly visible in the inset as a protrusion from the lower right of the disk. Their velocity difference is only 300 $\mathrm{km} / \mathrm{s}$, small enough to permit strong tidal interactions. 


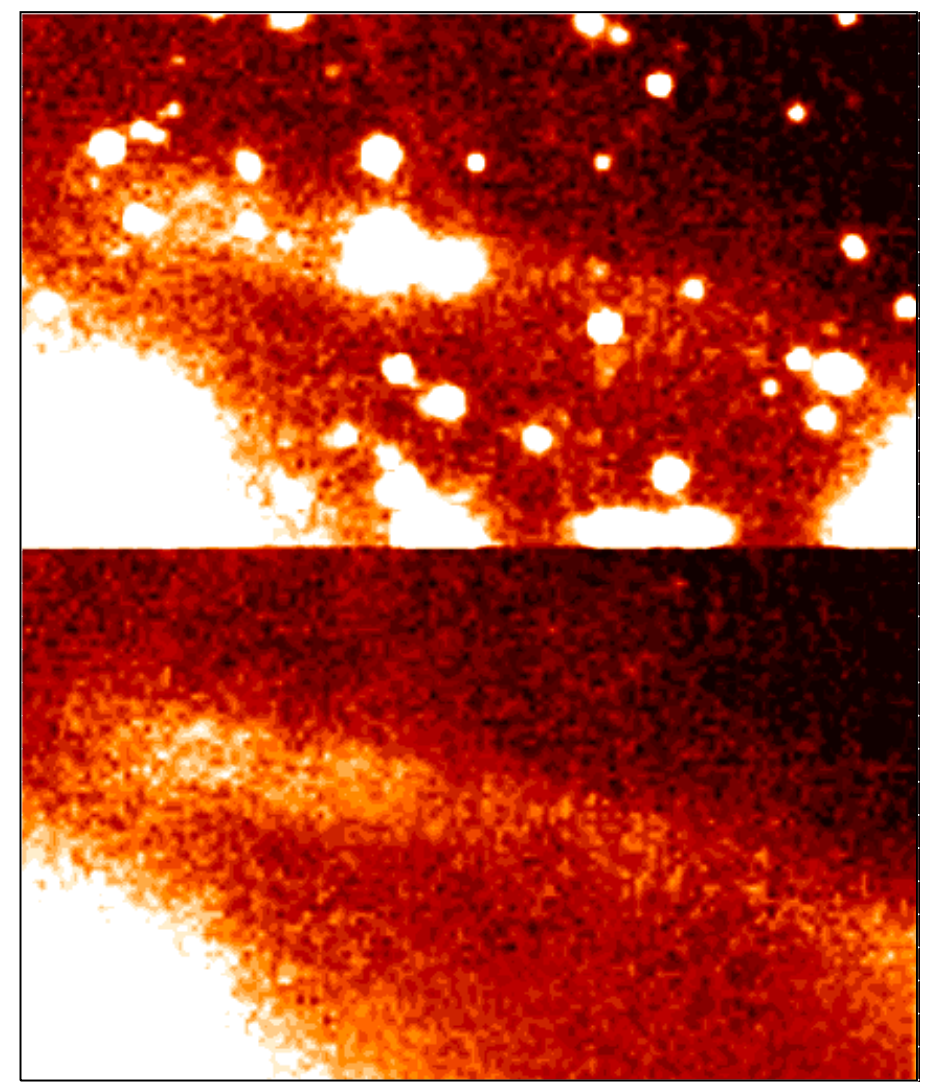




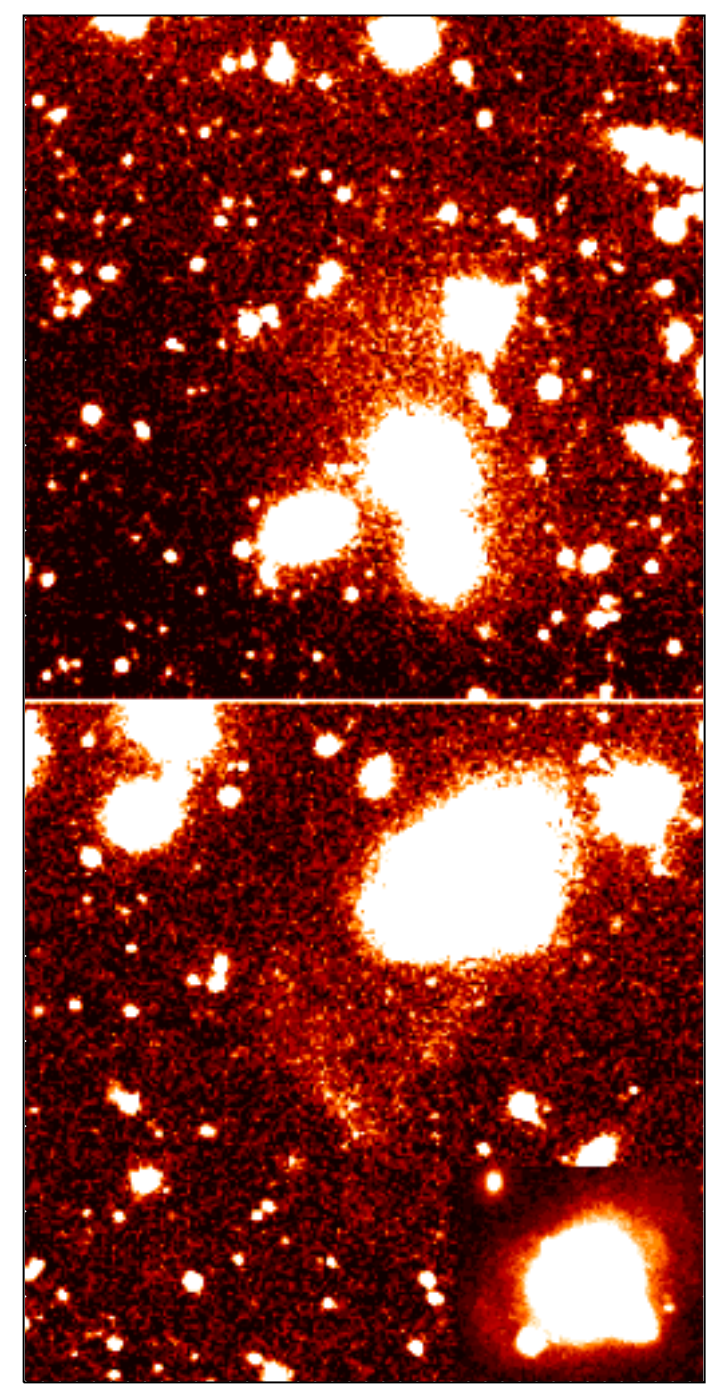

